\title{
Chest X-ray findings in a large cohort of 1117 patients with SARS-CoV-2 infection: a multicenter study during COVID-19 outbreak in Italy
}

\author{
Valentina Vespro ${ }^{1}$ (1) - Maria Carmela Andrisani ${ }^{1}$ - Stefano Fusco ${ }^{2} \cdot$ Letizia Di Meglio $^{2}$. Guido Plensich ${ }^{2}$. \\ Alice Scarabelli ${ }^{2}$. Elvira Stellato ${ }^{2}$. Anna Maria lerardi ${ }^{1}$. Luigia Scudeller ${ }^{3}$. Andrea Coppola ${ }^{4}$. Andrea Gori ${ }^{5}$. \\ Antonio Pesenti ${ }^{6}$. Giacomo Grasselli ${ }^{6}$. Stefano Aliberti ${ }^{7}$. Francesco Blasi ${ }^{7}$. Chiara Villa ${ }^{8}$. Sonia Ippolito ${ }^{8}$. \\ Barbara Pirrò $^{8}$. Guglielmo Damiani ${ }^{8} \cdot$ Massimo Galli $^{9} \cdot$ Giuliano Rizzardini $^{9} \cdot$ Emanuele Catena $^{10}$. \\ Matteo Agostino Orlandi ${ }^{11}$. Sandro Magnani ${ }^{11}$. Giuseppe Cipolla ${ }^{12}$. Andrea Antonio lanniello ${ }^{13}$. Mario Petrillo ${ }^{13}$. \\ Genti Xhepa ${ }^{13,14}$. Antonio Scamporrino ${ }^{14}$ - Alberto Cazzulani ${ }^{14}$. Gianpaolo Carrafiello ${ }^{1,15}$
}

Received: 5 July 2020 / Accepted: 31 October 2020 / Published online: 20 November 2020

(c) The Author(s) 2020

\begin{abstract}
To describe radiographic key patterns on Chest X-ray (CXR) in patients with SARS-CoV-2 infection, assessing the prevalence of radiographic signs of interstitial pneumonia. To evaluate pattern variation between a baseline and a follow-up CXR. 1117 patients tested positive for SARS-CoV-2 infection were retrospectively enrolled from four centers in Lombardy region. All patients underwent a CXR at presentation. Follow-up CXR was performed when clinically indicated. Two radiologists in each center reviewed images and classified them as suggestive or not for interstitial pneumonia, recording the presence of ground-glass opacity (GGO), reticular pattern or consolidation and their distribution. Pearson's $\chi^{2}$ test for categorical variables and McNemar test $\left(\chi^{2}\right.$ for paired data) were performed. Patients mean age 63.3 years, 767 were males $(65.5 \%)$. The main result is the large proportion of positive CXR in COVID-19 patients. Baseline CXR was positive in 940 patients (80.3\%), with significant differences in age and sex distribution between patients with positive and negative CXR. 382 patients underwent a follow-up CXR. The most frequent pattern on baseline CXR was the GGO (66.1\%), on follow-up was consolidation (53.4\%). The most common distributions were peripheral and middle-lower lung zone. We described key-patterns and their distribution on CXR in a large cohort of COVID-19 patients: GGO was the most frequent finding on baseline CXR, while we found an increase in the proportion of lung consolidation on follow-up CXR. CXR proved to be a reliable tool in our cohort obtaining positive results in $80.3 \%$ of the baseline cases.
\end{abstract}

Keywords COVID-19 $\cdot$ Radiography $\cdot$ Thoracic $\cdot$ Diagnosis $\cdot$ Pandemics

$\begin{array}{ll}\text { Abbreviations } & \\ \text { COVID-19 } & \text { Coronavirus disease 2019 } \\ \text { CT } & \text { Computed tomography } \\ \text { CXR } & \text { Chest X-ray } \\ \text { ED } & \text { Emergency department } \\ \text { GGO } & \text { Ground glass opacity } \\ \text { RT-PCR } & \text { Reverse transcription polymerase chain } \\ & \text { reaction }\end{array}$

SARS-CoV-2 Severe acute respiratory

syndrome-coronavirus-2

Valentina Vespro

valentina.vespro@policlinico.mi.it

Extended author information available on the last page of the article

\section{Introduction}

In late December 2019, local health authorities reported clusters of patients with pneumonia of unknown etiology, epidemiologically linked to a seafood market in Wuhan, Hubei Province, China [1].

A surveillance mechanism for "pneumonia of unknown etiology" was established with the aim of allowing timely identification of novel pathogens; the pathogen, a novel coronavirus (SARS-CoV-2), was identified. On 30 January 2020, the World Health Organization (WHO) declared that COVID-19 is a "public-health emergency of international concern" [2].

The pandemic has escalated rapidly; the first person-toperson transmission in Italy was reported on Feb 21st, 2020 
[3]. WHO data as of April 25th, 2020, reported 192,994 confirmed cases in Italy, with 25,969 deaths and a lethality rate of $13.5 \%$ [4].

The diagnosis and treatment program (7th version) published by the National Health Commission of the People's Republic of China no longer considers the radiologic features of lung involvement a diagnostic criteria for COVID19 [5]. Early discussions suggested that computed tomography (CT) should be the preferred modality for diagnosis of COVID-19, due to its higher sensitivity. However, the use of CT for COVID-19 diagnosis is controversial $[6,7]$.

In addition, in most hospitals, utilization of CT room for every patient with COVID-19 infection ascertained or suspected should be not easily manageable in terms of staff commitment, CT room workflow and disinfection procedures. Like any procedure performed on highly infective patients, it's mandatory to guarantee the effectiveness of the isolation itself, the safety of medical staff at risk.

Moreover, with the dramatic trend described above, early diagnosis of this newly emerging and life-threatening infection is crucial for the management of those patients, and therefore diagnostic procedures should be limited to the essential ones.

So far, radiological literature has primarily focused on CT findings, and a detailed overview of CXR appearance in relation to the disease is still poor. Therefore, the primary aim of our retrospective multicentric study was to describe the radiographic key patterns on Chest X-Ray (CXR) in patients with SARS-CoV-2 infection. In addition, we aimed at assessing the prevalence of individual radiographic signs of COVID-19 pneumonia among all the patients tested positive for SARS-CoV-2. Also, we compared patients with normal CXR vs those with at least one abnormal finding, in terms of demographic and clinical variables; finally, we estimated pattern variation between a baseline CXR and a follow-up CXR, to assess the utility of CXR in supporting the management of COVID-19 pneumonia.

\section{Materials and methods}

\section{Setting}

Four hospitals in Lombardy region participated to the study: three located in Milan area (ASST Fatebenefratelli Ospedale Luigi Sacco, Fondazione IRCCS Ca' Granda Ospedale Maggiore Policlinico, ASST Rhodense-Presidio Ospedaliero di Garbagnate Milanese), and one in Lodi (ASST LodiOspedale Maggiore di Lodi).

This retrospective cross-sectional study, with a longitudinal component, was conducted in accordance with the principles of the Declaration of Helsinki. Each institutional review board approved the study according to General Data Protection Regulation (GDPR).

\section{Patients}

All consecutive adult ( $>18$ years) patients referred to the Emergency Department (ED), with laboratory-identified SARS-CoV-2 infection by real time Reverse Transcription-Polymerase Chain Reaction (RT-PCR), were enrolled between February 22nd, 2020, and March 18th, 2020. All patients had at least one CXR at presentation.

The following clinical variables were extracted from patient's charts: age, sex, exposure history, comorbid conditions, symptoms.

A repeat CXR was performed in the admission ward, when clinically indicated by the attending physician.

\section{RT-PCR test}

Nasopharyngeal and oropharyngeal specimens collected with synthetic fiber swabs (manufactured by Copan) were laboratory tested with real time RT-PCR to detect SARSCoV-2 nucleotides.

The real time RT-PCR Tests were performed using GeneFinder ${ }^{\text {TM }}$ COVID-19 Plus RealAmp Kit manufactured by OSANG Healtcare (CE-IVD marked, fulfilling European Directive 98/79/EC) and Allplex ${ }^{\mathrm{TM}}$ 2019-nCoV Assay manufactured by Seegene (approved by Korean Food and Drug Administration).

\section{Imaging acquisition and interpretation}

The majority of CXR were performed bedside with portable digital radiographic equipment, owing to the impossibility to move the patient and/or to avoid his transportation from isolation areas. In the other cases CXR were acquired with patient standing with standard two projections.

Different digital radiographic equipment has been used: Ysio Max system and MOBILETT Elara Max mobile system (Siemens Healthcare) [Hospital A], Adora System (NRT $\mathrm{X}$-RAY A/S) and MAC mobile X-ray unit (General Medical Merate) [Hospital B], AXIOM Luminos dRF system (Siemens Healthcare) and FCR Go 2 portable system (Fujifilm) [Hospital C], FDR AcSelerate system and FDR Go PLUS portable system (Fujifilm) [Hospital D].

In each Hospital, two radiologists (with more than 10 years of experience in interpreting CXR imaging), reviewed all images, and classified CXR as suggestive or not suggestive for COVID-19 pneumonia. In case of disagreement, the same radiologists reviewed together the images to reach a consensus on the basis of the available radiological literature data. 
The epidemiological history and clinical symptoms (cough, fever, weakness, etc.) were available for both readers.

The presence of lung parenchymal abnormalities on each CXR was recorded in accordance with the Glossary of Terms for Thoracic Imaging of the Fleischner Society and defined as: (a) ground glass opacity (GGO); (b) lung consolidation; (c) reticular pattern [8].

GGO is defined as an area of hazy increased lung opacity, less opaque than consolidation, within which margins of pulmonary vessels may be indistinct (Fig. 1a, b). Consolidation is defined as a homogeneous increase in pulmonary parenchymal attenuation that obscures the margins of vessels and airway walls (Fig. 2a). Reticular pattern is
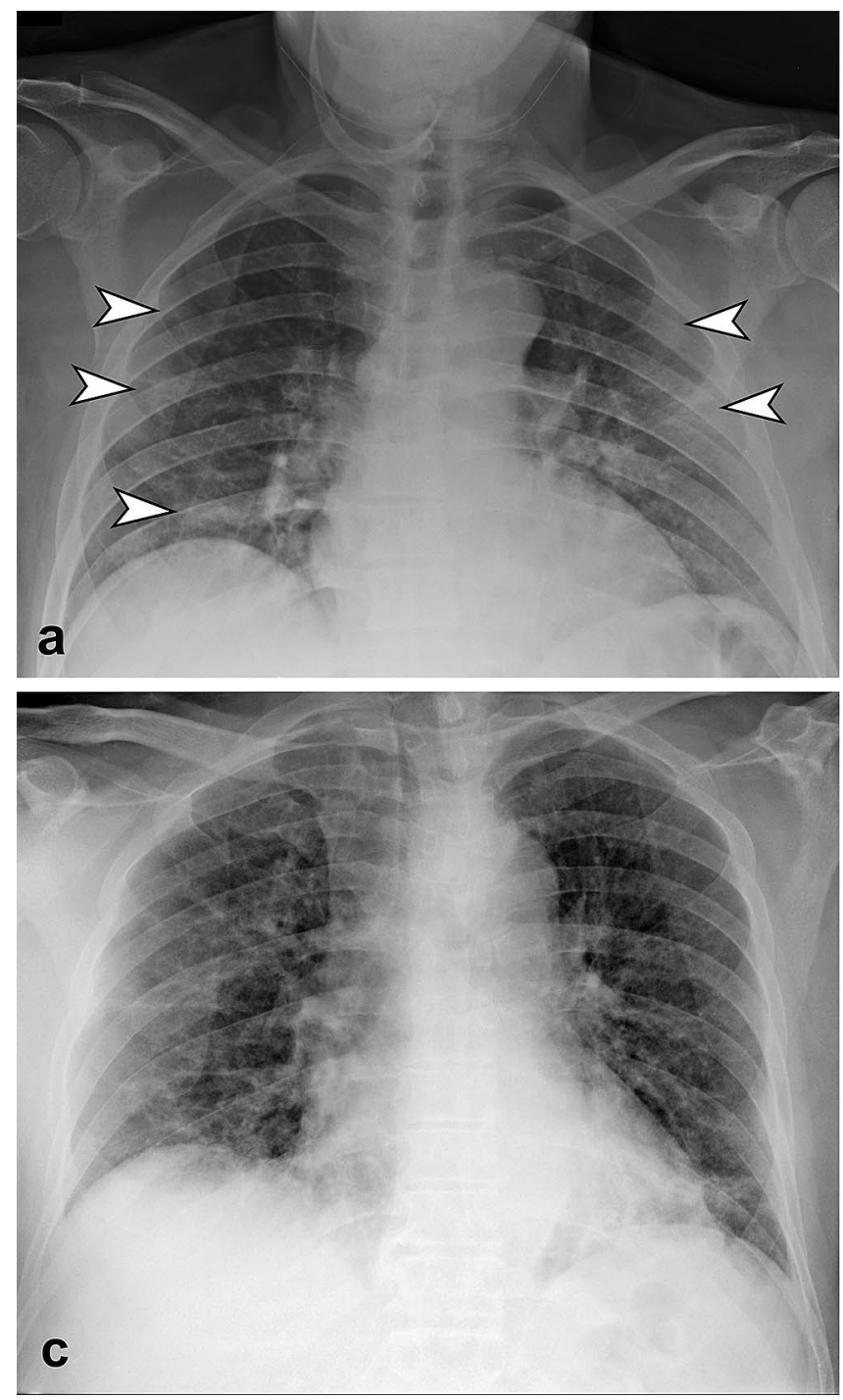

Fig. 1 a CXR in antero-posterior view shows bilateral ground glass opacities (arrowheads) with peripheral distribution, involving middlelower zone of the lungs. b Coronal reformatted CT image confirmed the CXR findings. c CXR in antero-posterior view shows bilateral defined as a collection of innumerable small linear opacities that, by summation, produce an appearance resembling a net (Fig. 1c, d, Fig. 2b).

Patients were considered to have findings suggestive for COVID-19 pneumonia if they had at least one of the above-mentioned features.

The distribution of the abnormal findings was recorded considering three criteria: (a) laterality (unilateral or bilateral); (b) axial distribution (central and/or peripheral); (c) longitudinal distribution (superior and/or middle and/or inferior).

Associated findings such as pleural effusion, pneumothorax and pneumomediastinum, were also recorded.
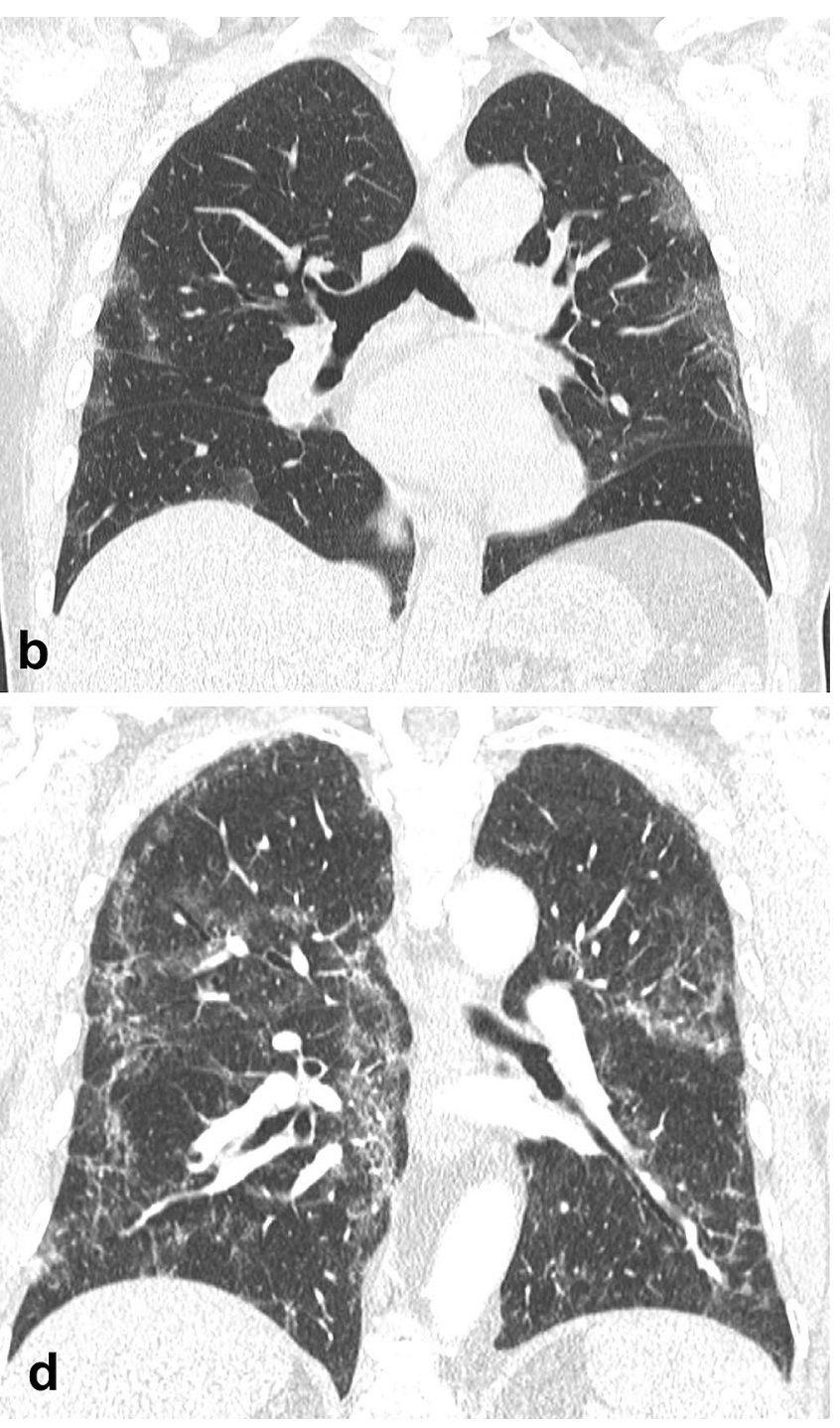

reticular pattern with diffuse distribution on both axial and longitudinal plane. d Coronal reformatted CT image confirms the presence of diffuse interstitial involvement with reticular pattern 


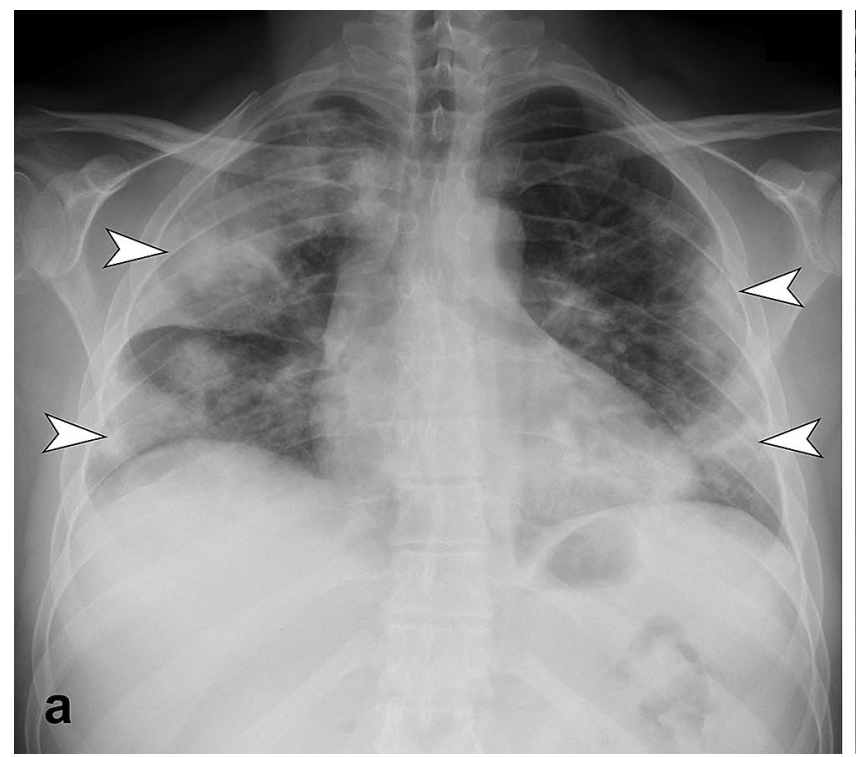

Fig. 2 a CXR in postero-anterior view shows bilateral multifocal consolidation (arrowheads), with greater involvement of the right lung. Consolidation present peripheral predominant distribution. b CXR in

In addition, on follow-up CXR radiologists evaluated the evolution of the radiographic findings describing if any improvement, a worsening or no significant changes occurred.

\section{Statistical analysis}

The study sample size was targeted at least 1000 patients, to be able to estimate proportions with precision at least $3.2 \%$. Descriptive statistics were produced for demographic, clinical and laboratory characteristics of cases. Mean and standard deviation (SD) are presented for normally distributed variables, and median and interquartile range (IQR) for non-normally distributed variables, number and percentages for categorical variables. Groups were compared with parametric or nonparametric tests, according to data distribution, for continuous variables, and with Pearson's $\chi^{2}$ test (Fisher exact test where appropriate) for categorical variables. Differences between first and second CXR were tested by means of the McNemar test ( $\chi^{2}$ for paired data). In all cases, 2 -tailed tests were used. $P$ value significance cutoff was 0.05. Stata computer software version 16.0 (Stata Corporation, 4905 Lakeway Drive, College Station, Texas 77845 , USA) was used for statistical analysis.

\section{Results}

The 1171 patients enrolled in our study had a mean age of 63.3 years (SD 15.9; range 18-96). There were 767 males (65.5\%) (Table 1). Fever was the most frequent symptom

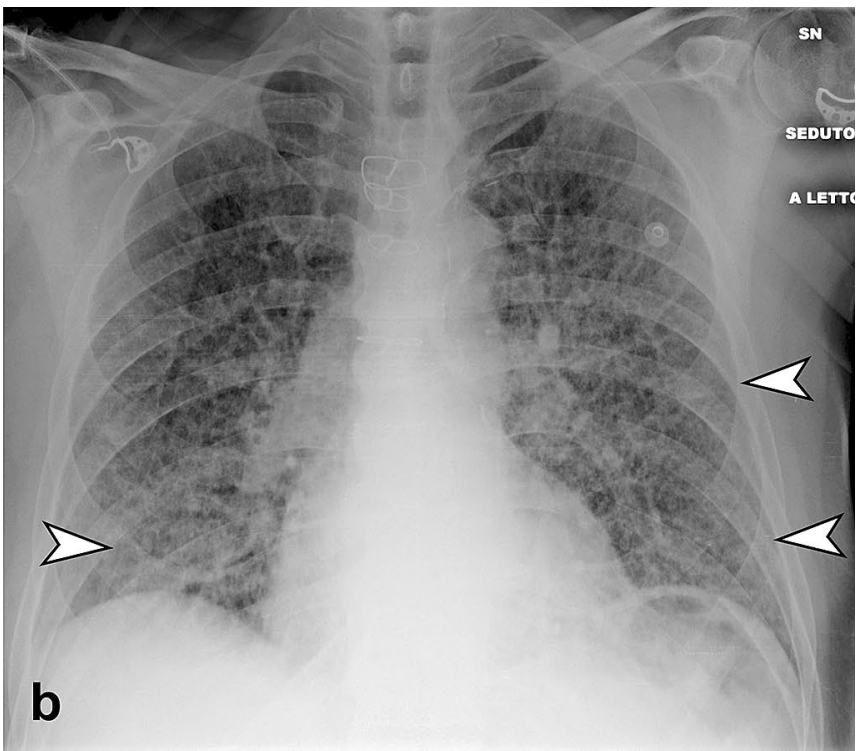

antero-posterior view shows the presence of diffuse bilateral reticular pattern. Note the compresence of bilateral subtle GGO (arrowheads) with peripheral and middle-lower predominant distribution

(79.9\%), while cough was present in $50.7 \%$ of the patients; 54 patients (4.6\%) were asymptomatic. The most common comorbidities were arterial hypertension (31.2\%), diabetes $(13.5 \%)$ and cardiovascular diseases (13.4\%).

CXR showed lung abnormalities in 940 patients $(80.3 \%)$. There were significant differences in age distribution between patients with positive (mean age: 65 years [SD 14.7]) and negative (56.6 years [SD 18.4] - $p$ value $<0.001])$ radiographic findings. Sex distribution also presented significant differences (positive CXR: 67.5\% males-32.5\% females; negative CXR: $57.6 \%$ males$42.4 \%$ females $-[p$ value $=0.005]$ )

The prevalence of fever and/or cough was similar among patients with positive CXR findings and patients with negative CXR, while dyspnea was present in $38.4 \%$ of patients with positive radiographic findings and in $21.7 \%$ of patients with negative radiographic findings ( $p$ value $<0.001)$.

\section{Radiographic findings on baseline CXR}

Of the 940 positive chest radiographs, 638 (67.9\%) showed the presence of a single pattern, while 273 (29\%) had the compresence of two patterns and $29(3.1 \%)$ presented three patterns.

The most frequent radiographic pattern, either isolated or combined, was the GGO (621/940, 66.1\%), followed by reticular pattern $(426 / 940,45.3 \%)$ and consolidation (224/940, 23.8\%). 
Table 1 Patient clinical characteristics

$\begin{array}{lll}\text { All patients }(n=1171) & \text { Patients with positive baseline } & \text { Patients with negative baseline } \\ & \operatorname{CXR}(n=940) & \operatorname{CXR}(n=231)\end{array}$
CXR $(n=940) \quad$ CXR $(n=231)$

\begin{tabular}{|c|c|c|c|c|}
\hline \multicolumn{5}{|l|}{ Characteristics } \\
\hline Mean age (years) & $63.3(\mathrm{SD} 15.9)$ & $65(\mathrm{SD} 14.7)$ & $56.6(\mathrm{SD} 18.4)$ & 0.001 \\
\hline \multicolumn{5}{|l|}{ Sex } \\
\hline Male & $767(65.5 \%)$ & $634(67.5 \%)$ & $133(57.6 \%)$ & 0.005 \\
\hline Female & $404(34.5 \%)$ & $306(32.55 \%)$ & $98(42.4 \%)$ & 0.005 \\
\hline \multicolumn{5}{|l|}{ Symptoms } \\
\hline Fever & $935(79.9 \%)$ & $739(78.6 \%)$ & $196(84.9 \%)$ & $<0.001$ \\
\hline Cough & $594(50.7 \%)$ & $476(50.6 \%)$ & $118(51.1 \%)$ & $<0.001$ \\
\hline Dyspnea & $411(35.1 \%)$ & $361(38.4 \%)$ & $50(21.7 \%)$ & $<0.001$ \\
\hline Sore throat & $25(2.1 \%)$ & $10(1 \%)$ & $15(6.5 \%)$ & - \\
\hline Diarrhea & $44(3.8 \%)$ & $38(4 \%)$ & $6(2.6 \%)$ & - \\
\hline Vomiting & $24(2 \%)$ & $21(2.2 \%)$ & $3(1.3 \%)$ & - \\
\hline Headache & $18(1.5 \%)$ & $12(1.3 \%)$ & $6(2.6 \%)$ & - \\
\hline Syncope & $32(2.7 \%)$ & $25(2.7 \%)$ & $7(3 \%)$ & - \\
\hline Asymptomatic & $54(4.6 \%)$ & $38(4 \%)$ & $16(6.9 \%)$ & $<0.001$ \\
\hline \multicolumn{5}{|l|}{ Comorbidities } \\
\hline Any & $692(59.1 \%)$ & $576(61.3 \%)$ & $116(50.2 \%)$ & - \\
\hline Hypertension & $365(31.2 \%)$ & $313(33.3 \%)$ & $52(22.5 \%)$ & - \\
\hline Diabetes & $158(13.5 \%)$ & $126(13.4 \%)$ & $32(13.9 \%)$ & - \\
\hline Cardiovascular disease & $157(13.4 \%)$ & $129(13.7 \%)$ & $28(12.1 \%)$ & - \\
\hline Pulmonary disease & $106(8.8 \%)$ & $78(8.3 \%)$ & $28(12.1 \%)$ & 0.001 \\
\hline Malignancy & $60(5.1 \%)$ & $58(6.2 \%)$ & $2(0.9 \%)$ & - \\
\hline
\end{tabular}

Peripheral $(524 / 940,55.8 \%)$ and middle-lower zone distribution $(331 / 940,33.1 \%)$ were the most common locations, and the $73.9 \%(695 / 940)$ had bilateral involvement.

Pleural effusion was described only in 7 cases.

The frequency of the three patterns and their distribution are listed in Table 2.

\section{Radiographic findings on follow-up CXR}

382 patients underwent a follow-up CXR. The median time between the two examinations was 4 days (IQR 3; range 1-17).

In 351 patients the first CXR examinations yielded positive results, confirmed on the second CXR.

The remaining 31 patients had normal findings on the first examination: the subsequent CXR showed radiographic abnormalities in 26 cases, while confirmed normal findings in 5 cases. Therefore, the overall prevalence of positive follow-up CXR was 98.7\% (377/382).

In $262 / 382$ cases $(68.6 \%)$ we noticed a worsening of the imaging findings, 73/382 (19.1\%) CXR were not significantly changed, while in $47 / 382(12.3 \%)$, cases there was an improvement.

The prevalence of the key patterns in the follow-up group changed as follows: consolidation was described in the $26 \%$ (99/382) of cases on the baseline CXR, while was present in the 53.4\% (204/382) on the follow-up CXR; on the other hand, the prevalence of GGO and reticular pattern decreased on the follow-up CXR, passing from $59.4 \%$ $(227 / 382)$ to $51 \%(195 / 382)$ and from $42.4 \%(162 / 382)$ to $37.2 \%$ (142/382), respectively (Fig. 3).

As seen above, the prevalence of consolidation in the follow-up group, increased significantly (from 26 to $53.4 \%-p$ value $<0.001)$; furthermore, 116 patients with no consolidation on the baseline CXR showed consolidation on the follow-up CXR, while only in 12 cases consolidation was seen on the first CXR and was no longer present at the follow-up.

Peripheral (48.1\%) and middle-lower zone distribution (40.2\%) remained the most common locations of the lung abnormalities, but we observed a greater involvement of the lung parenchyma both on axial and longitudinal distribution, in particular central-peripheral distribution was present in the $47.6 \%$ of patients (in contrast with $39.6 \%$ on the baseline CXR) and superior-middle-inferior distribution was described in the $27.9 \%$ of patients (in contrast with $16.8 \%$ on the baseline CXR). Moreover, the percentage of bilateral involvement of the lung increased from 76.6 to $87.7 \%$.

Other findings recorded were pleural effusion (6/382), pneumothorax (2/382) and pneumomediastinum (2/382). 
Table 2 Findings on positive baseline CXR $(n=940)$

\begin{tabular}{|c|c|}
\hline \multicolumn{2}{|l|}{ Patterns (alone or combined) } \\
\hline GGO & $621(66.1 \%)$ \\
\hline Reticular pattern & $426(45.3 \%)$ \\
\hline Consolidation/s & $224(23.8 \%)$ \\
\hline \multicolumn{2}{|l|}{ Number of patterns present } \\
\hline One & $638(67.9 \%)$ \\
\hline Two & $273(29 \%)$ \\
\hline Three & $29(3.1 \%)$ \\
\hline \multicolumn{2}{|l|}{ Pattern combinations } \\
\hline GGO & $363(38.6 \%)$ \\
\hline $\mathrm{GGO}+$ reticular pattern & $184(19.6 \%)$ \\
\hline Reticular pattern & $169(18 \%)$ \\
\hline Consolidations & $90(9.6 \%)$ \\
\hline GGO + consolidations & $45(4.8 \%)$ \\
\hline Reticular pattern + consolidations & $40(4.3 \%)$ \\
\hline $\mathrm{GGO}+$ reticular pattern + consolidations & $29(3.1 \%)$ \\
\hline Single consolidation & $16(1.7 \%)$ \\
\hline Single consolidation + reticular pattern & $4(0.4 \%)$ \\
\hline \multicolumn{2}{|l|}{ Axial distribution } \\
\hline Peripheral & $524(55.7 \%)$ \\
\hline Central & $311(33.1 \%)$ \\
\hline Diffuse (Central + peripheral) & $105(11.2 \%)$ \\
\hline \multicolumn{2}{|l|}{ Longitudinal distribution } \\
\hline Middle + inferior & $375(39.9 \%)$ \\
\hline Inferior & $208(22.1 \%)$ \\
\hline Superior + middle & $78(8.3 \%)$ \\
\hline Middle & $77(8.2 \%)$ \\
\hline Superior & $46(4.9 \%)$ \\
\hline Superior + inferior & $30(3.2 \%)$ \\
\hline Diffuse (Superior + middle + inferior) & $126(13.4 \%)$ \\
\hline \multicolumn{2}{|l|}{ Laterality } \\
\hline Unilateral & $245(26.1 \%)$ \\
\hline Bilateral & $695(73.9 \%)$ \\
\hline
\end{tabular}

The comparison between the findings on follow-up and baseline CXR is listed in Table 3.

\section{Discussion}

The main result of this study is the large proportion of positive CXR in patients with SARS-CoV-2 infection admitted to ED.

The most frequent lung abnormality detected on CXR was the GGO, present in $66.1 \%$ of our positive baseline CXR, confirming the data of the recent literature on both CXR and CT findings [6, 9-12].

Moreover, in line with the common CT findings of recent studies, the most frequent locations of lung abnormalities were the peripheral and middle-lower zone distribution, suggesting that the disease, may initially involve the parenchyma distal to the secondary lobule [13]. The bilateral involvement was also prevalent both in the baseline CXR (73.9\%) and in the follow-up CXR (85.9\%), consistent with other studies [6, 9, 14-16].

The reassessment of 382 patients with a follow-up CXR, has revealed an increase in the proportion of lung consolidation on positive CXR from about one fourth to over half. According to recent literature, this reflects the spectrum of diffuse alveolar damage (DAD) and/or organizing pneumonia, which tends to arise later in the disease course [14, 17, 18]. Furthermore, we were able to assess a greater extent of lung parenchymal involvement on follow-up radiographs. Since the repeat CXR was requested at discretion of the attending physician in case of suspected clinical worsening, these results suggest that there is an association between CXR findings evolution and the clinical progression of the disease $[11,14]$.

The vast majority of our patients were symptomatic and more than three quarters of our baseline CXR were positive. This data can be explained by the fact that, as opposed to what happened in Wuhan (China), where patients were encouraged to present to the hospital early in the course of their disease, in Italy the local authorities invited patients to stay at home until they would have experienced advanced symptoms [19, 20].

In such context, CXR often shows lung abnormalities and can be used as a first line assessment, while it is known that CXR has a low sensitivity in detecting early manifestations of COVID-19. Moreover it is important to be aware that even chest $\mathrm{CT}$ has been reported to demonstrate no abnormalities in the first 3 days of symptoms in 56\% of patients [21].

It is common knowledge that CXR has a lower sensitivity and specificity than CT. Nevertheless, due to the delicate management of high infectious patients, CXR has the considerable advantage of being conducted in the Biocontainment Unit (BU) or in isolating areas by mean of a portable digital radiographic equipment, thus reducing the risk of infection for healthcare workers and other patients. On the other hand, the use of a CT room for every patient with an ascertained or suspected SARS-CoV-2 infection must be carefully managed in terms of staff commitment, CT room workflow, disinfection procedures and consumption of personal protection equipment (PPE) [22]. Moreover, CXR also reduces the risk of radiation exposure to the patient.

As stated by the Multinational Consensus from the Fleischner Society, the choice of imaging modality should be subjected to the judgement of clinical teams taking into account the different properties of CXR and CT, the local resources and expertise [20].

Strengths of our study are the large sample size, unbiased representation of patients admitted to ED in our Region. However, our study presents several limitations. First of all, 
100

90

80

70

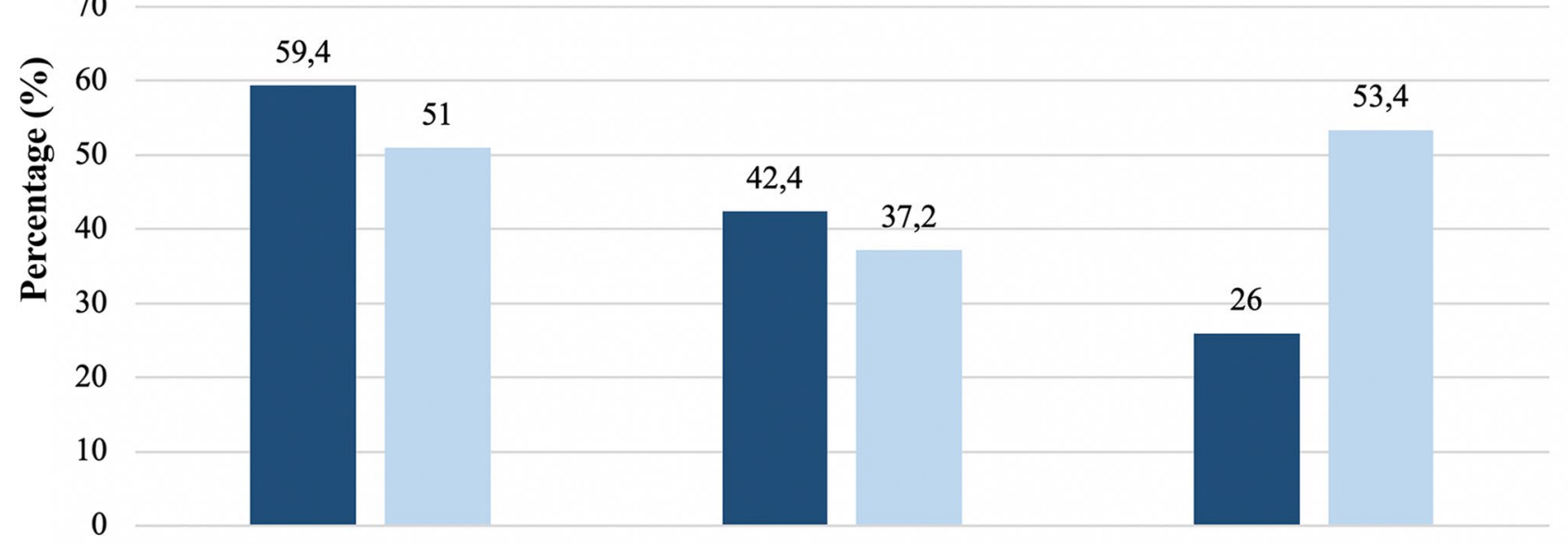

GGO

Reticular pattern

Consolidation/s

- Baseline CXR $\square$ Follow-up CXR

Fig. 3 Frequency of radiographic patterns on baseline and follow-up CXR $(n=382)$

Table 3 Comparison of findings in patient with baseline and follow-up CXR $(n=382)$

\begin{tabular}{lccc}
\hline & Baseline CXR & Follow-up CXR & $p$ value \\
\hline Positive & $351(91.2 \%)$ & $377(98.7 \%)$ & - \\
No abnormalities & $31(8.8 \%)$ & $5(1.3 \%)$ & - \\
Patterns (alone or combined) & & & $<0.001$ \\
GGO & $227(59.4 \%)$ & $195(51 \%)$ & 0.0181 \\
Reticular pattern & $162(42.4 \%)$ & $142(37.2 \%)$ & 0.0039 \\
Consolidation/s & $99(26 \%)$ & $204(53.4 \%)$ & $<0.001$ \\
Axial distribution & & \\
Peripheral & & $169(48.1 \%)$ & $<0.001$ \\
Central & $181(51.6 \%)$ & $15(4.3 \%)$ & \\
Diffuse (Central+Peripheral) & $32(9.1 \%)$ & $167(87.7 \%)$ & \\
Longitudinal distribution ${ }^{\mathrm{a}}$ & $138(76.6 \%)$ & $141(40.2 \%)$ & \\
Middle-inferior & & $37(10.5 \%)$ & \\
Inferior $_{\text {Superior-middle }}$ & $139(39.6 \%)$ & $33(9.4 \%)$ & \\
Middle & $60(17.1 \%)$ & $22(6.3 \%)$ & \\
Superior & $32(9.1 \%)$ & $7(2 \%)$ & \\
Superior-inferior & $29(8.3 \%)$ & $13(3.7 \%)$ & \\
Diffuse (superior-middle-inferior) & $21(6 \%)$ & $98(27.9 \%)$ & \\
Laterality $^{\mathrm{a}}$ & $11(3.1 \%)$ & $43(12.3 \%)$ & \\
Unilateral $^{\text {Bilateral }}$ & $59(16.8 \%)$ & $308(87.7 \%)$ & \\
\hline
\end{tabular}

N.B. the McNemar test for paired data was used (please see statistical methods)

${ }^{a}$ Percentages refer to patients with positive baseline CXR $(n=351)$ 
only $32.6 \%$ of our patients underwent a follow-up CXR. Secondly, we have limited clinical data due to the worldwide emergency situation; in particular we could not obtain information timing of symptoms onset. Thirdly, the interval between the baseline and the follow-up CXR is quite variable; also, we lack data about final patient outcomes. Moreover, we only included patients with CXR; however, the number of RT-PCR positive patients lacking CXR is estimated $<1 \%$, and this is unlikely to have caused bias. Finally, a control group with negative RT-PCR testing is lacking; however, we aimed at representing current clinical practice, where only patients with positive RT-PCR testing are diagnosed as proven infections.

It would be of strong clinical value to extend the study to all patients who access to ED with suspected SARS-CoV-2 infection, to evaluate the role of CXR in distinguishing patients with or without COVID-19.

In addition, it would be interesting to analyze the potential prognostic value of baseline CXR in predicting clinical outcome.

In conclusion, we have managed to describe the CXR key-patterns of COVID-19 and their distribution in a large cohort of patients during the peak of the COVID-19 outbreak in Italy. GGO was the most frequent finding on the baseline CXR, while we found an increase in the proportion of lung consolidation on the follow-up CXR. Despite being less sensitive than CT, CXR proved to be a reliable tool in our cohort obtaining positive results in $80.3 \%$ of the baseline cases.

Therefore, we believe that, in this specific epidemiological context, CXR should be the first line imaging technique. CT should be reserved to selected cases, e.g., in ruling out potential causes of acute symptoms worsening.

Funding Open access funding provided by Università degli Studi di Milano within the CRUI-CARE Agreement. The authors state that this work has not received any funding.

\section{Compliance with ethical standards}

Conflict of interest The authors of this manuscript declare no relationships with any companies, whose products or services may be related to the subject matter of the article.

Human and animal rights This retrospective cross-sectional study, with a longitudinal component, was conducted in accordance with the principles of the Declaration of Helsinki. Each institutional review board approved the study according to General Data Protection Regulation (GDPR)

Informed consent Written informed consent was waived by the Institutional Review Board.

Open Access This article is licensed under a Creative Commons Attribution 4.0 International License, which permits use, sharing, adaptation, distribution and reproduction in any medium or format, as long as you give appropriate credit to the original author(s) and the source, provide a link to the Creative Commons licence, and indicate if changes were made. The images or other third party material in this article are included in the article's Creative Commons licence, unless indicated otherwise in a credit line to the material. If material is not included in the article's Creative Commons licence and your intended use is not permitted by statutory regulation or exceeds the permitted use, you will need to obtain permission directly from the copyright holder. To view a copy of this licence, visit http://creativecommons .org/licenses/by/4.0/.

\section{References}

1. Zhu N, Zhang D, Wang W et al (2020) A novel coronavirus from patients with pneumonia in China, 2019. N Engl J Med 382:727-733

2. Li X, Wang W, Zhao X et al (2020) Transmission dynamics and evolutionary history of 2019-nCoV. J Med Virol 92:501-511

3. Spina S, Marrazzo F, Migliari M et al (2020) The response of Milan's Emergency Medical System to the COVID-19 outbreak in Italy. Lancet 395:e49-e50

4. WHO Coronavirus disease 2019 (COVID-19), Situation Report-96 (2020). https://www.who.int/docs/default-source/ coronaviruse/situation-reports/20200425-sitrep-96-covid-19. pdf? sfvrsn=a33836bb_2

5. National Health Commission of the People's Republic of China Diagnosis and Treatment Protocol for COVID-19 (Trial Version 7) (2020). https://en.nhc.gov.cn/2020-03/29/c_78469.htm

6. Chung M, Bernheim A, Mei X et al (2020) CT imaging features of 2019 Novel coronavirus (2019-nCoV). Radiology 295:202-207

7. ACR Recommendations for the use of Chest Radiography and Computed Tomography (CT) for Suspected COVID-19 Infection (2020). https://www.acr.org/Advocacy-and-Economics/ACR-Posit ion-Statements/Recommendations-for-Chest-Radiography-andCT-for-Suspected-COVID19-Infection

8. Hansell DM, Bankier AA, MacMahon H et al (2008) Fleischner society: glossary of terms for thoracic imaging. Radiology 246:697-722

9. Wong HYF, Lam HYS, Fong AH-T et al (2020) Frequency and distribution of chest radiographic findings in COVID-19 positive patients. Radiology. https://doi.org/10.1148/radiol.2020201160

10. Song F, Shi N, Shan F et al (2020) Emerging 2019 novel coronavirus (2019-nCoV) pneumonia. Radiology 295:210-217

11. Pan F, Ye T, Sun P et al (2020) Time course of lung changes on chest CT during recovery from 2019 novel coronavirus (COVID19) pneumonia. Radiology. https://doi.org/10.1148/radiol.20202 00370

12. Yang W, Sirajuddin A, Zhang X et al (2020) The role of imaging in 2019 novel coronavirus pneumonia (COVID-19). Eur Radiol. https://doi.org/10.1007/s00330-020-06827-4

13. Ding X, Xu J, Zhou J, Long Q (2020) Chest CT findings of COVID-19 pneumonia by duration of symptoms. Eur J Radiol 127:109009

14. Shi H, Han $X$, Jiang $N$ et al (2020) Radiological findings from 81 patients with COVID-19 pneumonia in Wuhan, China: a descriptive study. Lancet Infect Dis 20:425-434

15. Zhou S, Wang Y, Zhu T, Xia L (2020) CT features of coronavirus disease 2019 (COVID-19) pneumonia in 62 patients in Wuhan, China. Am J Roentgenol. https://doi.org/10.2214/AJR.20.22975 
16. Ng M-Y, Lee EY, Yang J et al (2020) Imaging profile of the COVID-19 infection: radiologic findings and literature review. Radiol Cardiothorac Imaging. https://doi.org/10.1148/ryct.20202 00034

17. Pan Y, Guan H, Zhou S et al (2020) Initial CT findings and temporal changes in patients with the novel coronavirus pneumonia (2019-nCoV): a study of 63 patients in Wuhan, China. Eur Radiol. https://doi.org/10.1007/s00330-020-06731-x

18. Bernheim A, Mei X, Huang $M$ et al (2020) Chest CT findings in coronavirus disease-19 (COVID-19): relationship to duration of infection. Radiology. https://doi.org/10.1148/radiol.2020200463

19. Ai T, Yang Z, Hou H et al (2020) Correlation of chest CT and RTPCR testing in coronavirus disease 2019 (COVID-19) in China: a report of 1014 cases. Radiology. https://doi.org/10.1148/radio 1.2020200642

20. Rubin GD, Haramati LB, Kanne JP et al (2020) The role of chest imaging in patient management during the COVID-19 pandemic: a multinational consensus statement from the Fleischner Society. Radiology. https://doi.org/10.1148/radiol.2020201365

21. Revel M-P, Parkar AP, Prosch H et al (2020) COVID-19 patients and the radiology department-advice from the European Society of Radiology (ESR) and the European Society of Thoracic Imaging (ESTI). Eur Radiol. https://doi.org/10.1007/s00330-02006865-y

22. Mossa-Basha M, Meltzer CC, Kim DC et al (2020) Radiology department preparedness for COVID-19: radiology scientific expert panel. Radiology. https://doi.org/10.1148/radiol.20202 00988

Publisher's Note Springer Nature remains neutral with regard to jurisdictional claims in published maps and institutional affiliations.

\section{Affiliations}

Valentina Vespro ${ }^{1}$ - Maria Carmela Andrisani ${ }^{1} \cdot$ Stefano Fusco ${ }^{2} \cdot$ Letizia Di Meglio $^{2}$. Guido Plensich ${ }^{2}$. Alice Scarabelli ${ }^{2}$. Elvira Stellato ${ }^{2}$ - Anna Maria lerardi ${ }^{1}$ - Luigia Scudeller ${ }^{3} \cdot$ Andrea Coppola $^{4}$ - Andrea Gori ${ }^{5}$. Antonio Pesenti ${ }^{6}$. Giacomo Grasselli ${ }^{6}$. Stefano Aliberti ${ }^{7}$. Francesco Blasi ${ }^{7}$. Chiara Villa ${ }^{8}$. Sonia Ippolito ${ }^{8}$. Barbara Pirrò $^{8}$. Guglielmo Damiani ${ }^{8} \cdot$ Massimo Galli $^{9} \cdot$ Giuliano Rizzardini $^{9} \cdot$ Emanuele Catena $^{10}$. Matteo Agostino Orlandi ${ }^{11}$. Sandro Magnani ${ }^{11}$. Giuseppe Cipolla ${ }^{12}$. Andrea Antonio lanniello ${ }^{13}$. Mario Petrillo ${ }^{13}$. Genti Xhepa ${ }^{13,14}$ - Antonio Scamporrino ${ }^{14}$ - Alberto Cazzulani ${ }^{14}$. Gianpaolo Carrafiello ${ }^{1,15}$

1 Department of Radiology, Fondazione IRCCS Ca' Granda Ospedale Maggiore Policlinico, University of Milan, Milan, Italy

2 Postgraduate School of Diagnostic and Interventional Radiology, University of Milan, Milan, Italy

3 Scientific Direction, Clinical Epidemiology and Biostatistics, Fondazione IRCCS Ca' Granda Ospedale Maggiore Policlinico, Milan, Italy

4 Department of Radiology, Università degli Studi dell'Insubria, Varese, Italy

5 Infectious Diseases Unit, Fondazione IRCCS Ca' Granda Ospedale Maggiore Policlinico, University of Milan, Milan, Italy

6 Department of Anesthesiology and Intensive Care Unit, Fondazione IRCCS Ca' Granda Ospedale Maggiore Policlinico, University of Milan, Milan, Italy

7 Department of Pathophysiology and Transplantation, University of Milan, Milan, Italy
8 Department of Radiology, Luigi Sacco Hospital, ASST Fatebenefratelli Sacco, University of Milan, Milan, Italy

9 Division of Infectious Diseases, Luigi Sacco Hospital, ASST Fatebenefratelli Sacco, University of Milan, Milan, Italy

10 Department of Anesthesiology and Intensive Care Unit, Luigi Sacco Hospital, ASST Fatebenefratelli Sacco, University of Milan, Milan, Italy

11 Department of Radiology, ASST Lodi, Ospedale Maggiore di Lodi, Lodi, Italy

12 Department of Respiratory Diseases, ASST Lodi, Ospedale Maggiore di Lodi, Lodi, Italy

13 Department of Radiology, ASST Rhodense, Garbagnate Milanese, Milan, Italy

14 CERBA HealthCare Italia, Cologno Monzese, Monza Brianza, Italy

15 Department of Health Science, University of Milan, Milan, Italy 